\title{
High resolution mapping of modified DNA nucleobases using excision repair enzymes
}

\author{
D. Suzi Bryan, ${ }^{1}$ Monica Ransom, ${ }^{1}$ Biniam Adane, Kerri York, and Jay R. Hesselberth \\ Department of Biochemistry and Molecular Genetics, Program in Molecular Biology, University of Colorado School of Medicine, Aurora, \\ Colorado 80045, USA
}

\begin{abstract}
The incorporation and creation of modified nucleobases in DNA have profound effects on genome function. We describe methods for mapping positions and local content of modified DNA nucleobases in genomic DNA. We combined in vitro nucleobase excision with massively parallel DNA sequencing (Excision-seq) to determine the locations of modified nucleobases in genomic DNA. We applied the Excision-seq method to map uracil in E. coli and budding yeast and discovered significant variation in uracil content, wherein uracil is excluded from the earliest and latest replicating regions of the genome, possibly driven by changes in nucleotide pool composition. We also used Excision-seq to identify sites of pyrimidine dimer formation induced by UV light exposure, where the method could distinguish between sites of cyclobutane and 6-4 photoproduct formation. These UV mapping data enabled analysis of local sequence bias around pyrimidine dimers and suggested a preference for an adenosine downstream from 6-4 photoproducts. The Excision-seq method is broadly applicable for high precision, genome-wide mapping of modified nucleobases with cognate repair enzymes.
\end{abstract}

[Supplemental material is available for this article.]

Many different modifications of the four primary DNA nucleobases expand the chemical diversity of DNA and have profound effects on genome function. Intrinsic modifications (e.g., 5methylcytosine and uracil) are integral to genetic and epigenetic regulation. Extrinsic modifications (e.g., pyrimidine dimers and nucleobase oxidation) arise from environmental exposures and can initiate aberrant cell growth or death. A detailed understanding of intrinsic and extrinsic nucleobase modification is necessary for a complete view of genetic and epigenetic regulation, but a global picture of how nucleobase modifications are created, maintained, and repaired, and how their spatial distribution impacts genome function, is lacking.

Incorporation of uracil into DNA creates detrimental or beneficial mutations, depending on context. To sustain DNA replication, cells must synthesize or scavenge precursors to accumulate a pool of nucleotide triphosphates. A key step of thymidine triphosphate (TTP) synthesis is catalyzed by thymidylate synthase, which converts dUMP to dTMP using tetrahydrofolate as a methyl donor. One branch of the TTP biosynthetic pathway uses dUTP as an intermediate, which can be incorporated into DNA in the form of A:U base pairs. The upstream production of dUMP is catalyzed by deamination of dCMP by deoxycytidylate deaminase or pyrophosphorolysis of dUTP by the dUTP pyrophosphatase (Dut1). Dut1 is essential for viability and normal nucleotide metabolism: In the absence of Dut1, cells simultaneously accumulate dUTP and deplete TTP pools (Gadsden et al. 1993), causing a futile cycle of uracil incorporation and repair that leads to extensive DNA damage (Kavli et al. 2007).

Uracil in DNA is removed by uracil DNA glycosylase (UDG) enzymes, which scan double-stranded DNA for uracil and cleave its glycosidic bond (Krokan et al. 2002; Kavli et al. 2007). Simultaneous

\footnotetext{
'These authors contributed equally to this work.
} Corresponding author: jay.hesselberth@ucdenver.edu Article published online before print. Article, supplemental material, and publication date are at http://www.genome.org/cgi/doi/10.1101/gr.174052.114. Freely available online through the Genome Research Open Access option. inactivation of dUTPase and uracil DNA glycosylase in E. coli results in viable cells that accumulate significant amounts of uracil in their DNA due to increased dUTP levels and an inability to remove uracil from DNA (Warner et al. 1981). Mutation of the dUTPase and uracil DNA glycosylase genes has also been used to cause uracil incorporation in S. cerevisiae (Guillet et al. 2006) and C. elegans (Dengg et al. 2006). Regions of high transcription exhibit elevated dUTP incorporation, suggesting that levels of UTP needed to sustain transcription can be converted to dUTP for incorporation into DNA (Kim and Jinks-Robertson 2009).

Pharmacologic treatments and metabolic imbalances also promote dUTP incorporation into DNA. Thymidylate synthase is a major target of anti-metabolite drugs, such as pemetrexed and 5fluorouracil, which inhibit TTP production by thymidylate synthase and simultaneously increase dUMP and dUTP levels (Longley et al. 2003). Because thymidylate synthase uses tetrahydrofolate as a methyl donor, folate deficiency also alters the pool of available nucleoside precursors for TTP synthesis, and phenocopies antifolate therapy by increasing the cellular levels of dUTP, causing its incorporation into DNA and subsequent chromosome instability (Blount et al. 1997).

Uracil in DNA also plays a prominent role in adaptive immunity. In B cells, somatic hypermutation and class switch recombination are mediated by the deamination of cytosine residues to uracil by the activation-induced cytosine deaminase (AICDA, also known as AID). AID is recruited to immunoglobulin loci and promotes somatic hypermutation by pseudo-randomly deaminating cytosines to uracil, which are repaired by error-prone polymerases, creating diversity (Maul and Gearhart 2010). Aberrant AID localization can promote hypermutation of tumor suppressor genes (Klemm et al. 2009) and initiates chromosome translocations via cytosine deamination and base excision repair. Signatures of uracilmediated mutations are widespread among cancer subtypes, and

(C) 2014 Bryan et al. This article, published in Genome Research, is available under a Creative Commons License (Attribution 4.0 International), as described at http://creativecommons.org/licenses/by/4.0. 
dysregulation of APOBEC family cytidine deaminases was implicated as the cause of these mutations (Alexandrov et al. 2013).

DNA exposure to ultraviolet light creates adducts like cyclobutane dimers and 6-4 photoproducts, formed between adjacent pyrimidines (Pfeifer 1997). Some organisms encode photolyase enzymes that recognize pyrimidine dimers and convert them to "monopyrimidines" in an FAD-dependent reaction using visible light. In the absence of photolyase repair, pyrimidine dimers are excised by nucleotide excision repair enzymes, some of which are mutated in human diseases such as xeroderma pigmentosum, in which UV light creates damage that cannot be repaired (Friedberg 2001). In addition, some translesion DNA polymerases are capable of incorporation across from a pyrimidine dimer in the template strand (Sale 2013).

Pyrimidine dimer formation and prevalence in DNA is dictated by several factors including CpG methylation status, dinucleotide content, and chromatin context (Smerdon and Conconi 1999; Law et al. 2013; Zavala et al. 2014). Moreover, repair of UV photoproducts is strongly influenced by genomic context. Photoproducts formed on transcribed template strands are repaired much more efficiently than nontemplate strands by transcription-coupled repair (Hanawalt and Spivak 2008). In addition, some hot spots of pyrimidine dimer formation are repaired efficiently, whereas other sites are more likely to yield mutations (Tornaletti et al. 1994; You et al. 2000). However, studies of pyrimidine dimer formation in the human genome have been restricted to specific loci (Pfeifer et al. 1991; Törmänen and Pfeifer 1992) or indirectly monitor pyrimidine dimer repair on transfected DNA substrates (Protić-Sabljić et al. 1986), yielding a narrow view of pyrimidine dimer formation and repair in large genomes.

Some methods provide information about specific sites of modified bases in a genome, but have limited applicability because of the need to interrogate a region of interest by PCR. For example, ligation-mediated PCR (LM-PCR) maps nucleobase modifications by ligating PCR-competent adaptors to the ends of cDNA created by termination of polymerase elongation in vitro when it encounters nicks and single base gaps introduced by DNA modification (Sugasawa 2010; Yan et al. 2011; Besaratinia and Pfeifer 2012). LMPCR has been used to map UV modification using base excision repair enzymes coupled with gene specific probes (Sugasawa 2010; Yan et al. 2011; Besaratinia and Pfeifer 2012) and has also been used to map uracil in the context of AID-mediated deamination (Maul et al. 2011).

Other methods provide global views of nucleobase modification but suffer from low resolution or scalability. A number of methods have been developed to examine DNA methylation and demethylation by chemical modification or affinity purification of 5-methylcytosine and 5-hydroxymethylcytosine (Wu and Zhang 2014). These methods can provide single-nucleotide resolution measures of DNA methylation and demethylation, but they have not been widely applied due to the cost of whole genome coverage needed for accurate determination of sites of chemical modification. Microarray approaches have been used to survey DNA damage caused by ultraviolet light and methylation throughout the genome, using antibodies with an affinity for specific modified nucleobases or enzymes that recognize and cleave modification nucleobases (Schumacher et al. 2006; Zavala et al. 2014). These methods achieve resolution in the 100-1000 base pair range, precluding direct identification of specific sites of DNA modification. A new method based on ChIP-seq for mapping sites of singlestranded DNA enables global studies of putative DNA damage but does not identify specific modified nucleobases (Zhou et al. 2013).
Finally, new single-molecule sequencing platforms detect a variety of modified nucleobases in their native contexts (Clarke et al. 2009; Clark et al. 2011; Kozdon et al. 2013), but nanopore sequencers are not yet widely available, and real-time analysis of single molecule polymerase incorporation events suffers from a high error rate and high cost of genome-wide coverage, limiting comprehensive characterization of large eukaryotic genomes.

Here we developed a method that couples the specificity and efficiency of excision repair enzymes with the scale and throughput of massively parallel DNA sequencing to identify single sites or local content of modified nucleobases throughout the genome. This new Excision-seq method provides advantages over existing methods by generating sequencing libraries that are enriched for sites of modification, while maintaining high resolution mapping information. The Excision-seq method is applicable to the detection of DNA modifications for which cognate repair enzymes are available.

\section{Results}

\section{Development of Excision-seq}

We developed methods to map modified nucleobases in genomic DNA with high precision by coupling base and nucleotide excision repair enzymes with next-generation DNA sequencing (Excisionseq). Excision-seq comprises two related approaches that reflect the point at which modified nucleobases are removed: In "predigestion" Excision-seq (Fig. 1A), a base excision repair enzyme removes modified bases in genomic DNA, creating abasic sites that are removed with $\mathrm{T} 4$ endonuclease IV, leaving a single base gap. When the modified base is present in high abundance, this treatment releases small double-stranded DNA fragments that can be converted into libraries suitable for next-generation DNA sequencing. Importantly, the ends of these fragments correspond to the previous locations of the modified base, allowing their identification by sequencing. The predigestion approach precisely identifies positions of modified base incorporation. One drawback of predigestion Excision-seq is that high levels of the modified base are required to yield fragments upon digestion for subsequent library construction. To address this limitation, we developed a complementary approach termed "post-digestion" Excision-seq (Fig. 1B), in which genomic DNA is sheared and converted to a sequencing library by end polishing and adaptor ligation. Prior to PCR, the library is treated with a base excision repair enzyme to destroy one or both strands from each double-stranded DNA in the library that contains modified bases. Undigested strands are amplified and sequenced to identify regions in which the modified nucleobases were not present. Post-digestion Excision-seq is useful when modified bases are excluded from regions of the genome or when the abundance of the modified bases is insufficient to yield sufficient double-strand breaks needed for the predigestion method. The post-digestion method provides information about the content of modified nucleobases and can achieve resolution that correlates with fragment lengths used to build the sequencing library.

\section{Application of Excision-seq to study uracil in DNA}

We used predigestion Excision-seq to map the locations of uracil in the $E$. coli genome. We digested genomic DNA from E. coli with a hypomorphic dUTPase that was also missing the uracil repair enzyme (Warner et al. 1981) in vitro with uracil DNA glycosylase (UDG) and T4 Endonuclease IV (Fig. 1C), yielding DNA fragments that were converted to a sequencing library. In parallel, we prepared 
A

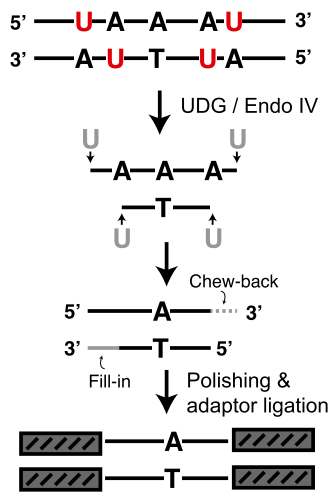

C

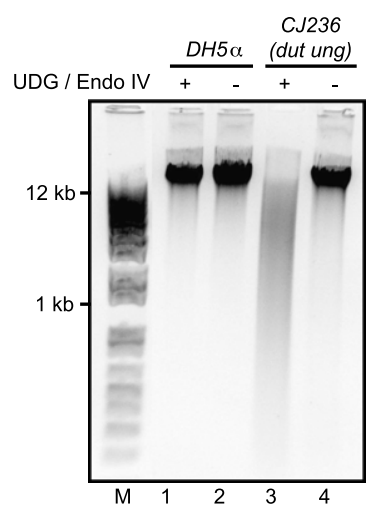

B

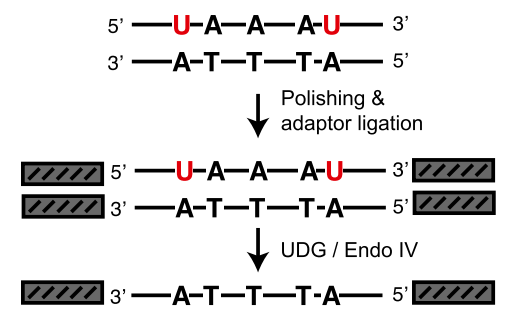

D

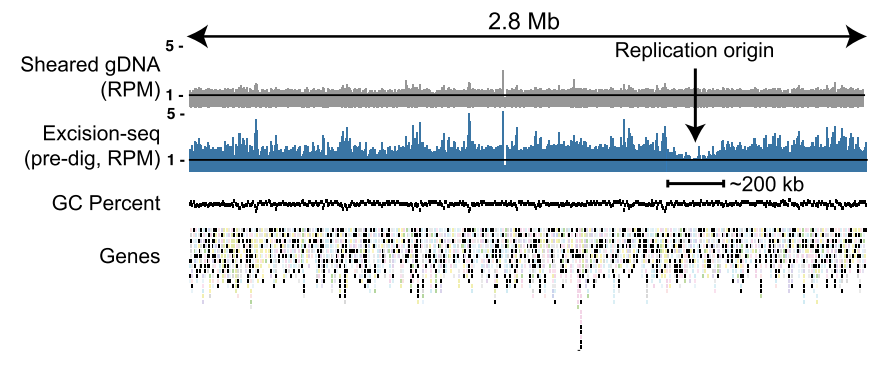

Figure 1. Excision-seq methods for mapping modified nucleobases in genomic DNA. (A) In "predigestion" Excision-seq for uracil, uracil-containing DNA is cut with a base excision repair enzyme (e.g., E. coli UDG, red). Released fragments are end-repaired, A-tailed, ligated to adaptors, and PCR amplified. Sequences derived from this library identify the positions of modified bases (e.g., one base upstream of the $5^{\prime}$-most position of the read). (B) In "post-digestion" Excision-seq for uracil, DNA is sheared mechanically, then treated by standard polishing and ligation. A base excision enzyme cleaves one or both strands containing modified bases. Intact strands remaining after digestion are PCR amplified and sequenced. (C) Genomic DNA isolated from dut ung E. coli is digested by UDG and T4 endonuclease IV (cf. lanes 3 and 4), whereas genomic DNA isolated from a wild-type strain is not digested (lanes 1 and 2). (D) Normalized coverage from shotgun sequencing of mechanically sheared genomic DNA (gray, reads per million [RPM]) and predigestion Excisionseq for uracil (blue, RPM) for a 2.8-Mb region of the E. coli chromosome. GC-content and the positions of protein-coding genes are plotted below. Uracil content is lowest in a region centered on the origin of replication, encompassing $\sim 200 \mathrm{~kb}$ of DNA.

a library from mechanically sheared DNA to test whether uracil content affects library preparation efficiency. We collected and aligned $\sim 10$ million sequences from these libraries and found that the library prepared from genomic DNA exhibited uniform coverage across the genome (Fig. 1D). The low coverage of reads from the Excision-seq library in the $\sim 200$-kb region surrounding the replication origin unexpectedly suggested low levels of uracil incorporation in this region of the genome (Fig. 1D).

To further study this phenomenon, we used pre- and postdigestion Excision-seq to map uracil in a dut1-1 ung1 $\Delta$ budding yeast strain (Guillet et al. 2006). We examined the frequency of bases surrounding the mapped positions of reads in each library. In predigestion Excision-seq libraries for mapping uracil, we expected to find that the base upstream of mapped positions would correspond to a Tresidue, representing the previous location of a uracil (Fig. 1A). More than $98 \%$ of reads from uracil Excision-seq libraries mapped to positions downstream from $\mathrm{T}$ residues (Fig. 2A), indicating robust recovery of uracil sites. In post-digestion Excision-seq libraries for uracil, we expected a more random distribution of base identity at the sites of linker ligation (Fig. 1B) and found relatively uniform levels of base identity near the sites of linker ligation (Fig. 2B).

Given the low uracil content at the E. coli replication origin, we considered whether uracil content in S. cerevisiae would correlate with replication timing. We quantified the relationship between uracil content and replication timing (Raghuraman et al. 2001; Yabuki et al. 2002) by calculating the coverage in post-digestion Excision-seq uracil libraries in 500-bp windows, and compared the coverage to replication timing (Fig. 2C). We found that uracil content was lowest (e.g., highest post-digestion signals) in early-replicating regions. Uracil content increased steadily over the first $\sim 8 \mathrm{~min}$ of $S$ phase and remained constantly high until uracil content decreased again within the last $10 \mathrm{~min}$ of $\mathrm{S}$ phase. As a control, we also calculated AT content over the same intervals and found no significant variation in AT content across these regions (Fig. 2D), confirming that uracil content is not driven by variation in genomic nucleotide content. In the E. coli predigestion Excision-seq mapping of uracil, we identified local correlation between uracil content and local GC content at a size range of 1-10 bp; this bias was not apparent in the $S$. cerevisiae mapping data. Thus, whereas there is some relationship between uracil content and genomic AT content, the variation in uracil content that we observe changes over the kilobase scale, far greater than local changes in AT content.

Visualization of Excision-seq mapping data for uracil at the chromosomal level reinforced a correlation between replication timing and uracil content. A total of 42 early-firing origins were depleted of uracil, (e.g., ARS418 and ARS428) (Fig. 3A-C,E). In

\section{Genome Research} www.genome.org 
A

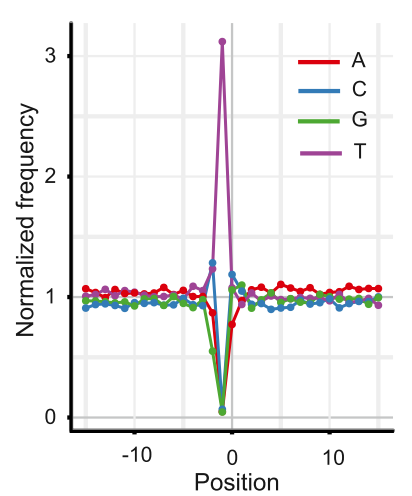

C

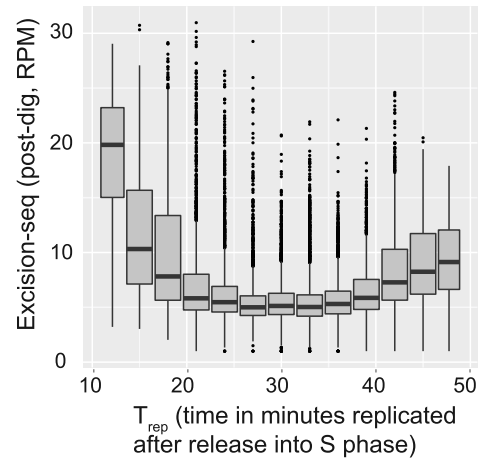

B

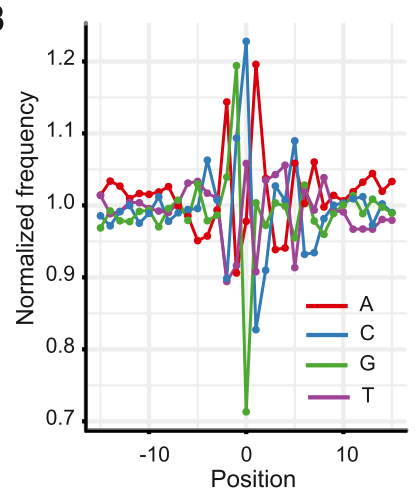

D

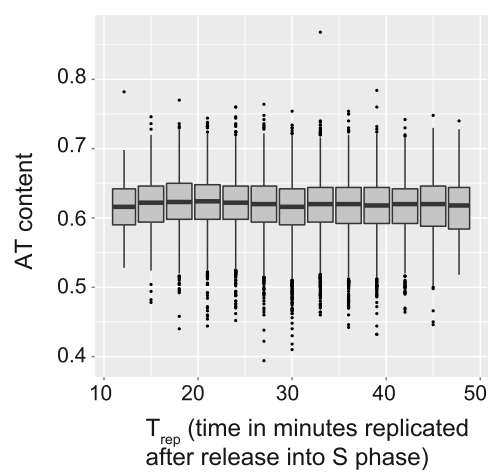

Figure 2. Excision-seq mapping of uracil content in the budding yeast genome. $(A)$ Normalized frequency of nucleotides relative to mapped positions of sequences from predigestion Excision-seq libraries for mapping S. cerevisiae uracil content. Position 0 corresponds to the mapped position of the 5 end of 11,326,044 sequencing reads; negative numbers are upstream. Frequencies were normalized to genomic mononucleotide frequencies. (B) Normalized frequency of nucleotides relative to mapped positions of sequences from post-digestion Excision-seq libraries for mapping S. cerevisiae uracil content. Position 0 corresponds to the mapped position of the $5^{\prime}$ end of 2,939,357 sequencing reads (frequency normalization and colors identical to $A$ ). (C) Boxplot comparing post-digestion Excision-seq mapping of uracil in S. cerevisiae to replication timing ( $T_{\text {rep }}$ ) (Raghuraman et al. 2001). Mean signals were calculated in 500-bp windows across the genome. (D) Boxplot comparing mean genomic AT content in S. cerevisiae to replication timing; 500-bp regions are identical to $C$.

addition, late-replicating regions exhibit a significant but more modest depletion of uracil (Fig. 3A,B). Sequence read coverage in post-digestion Excision-seq data is inversely proportional to content of the modified nucleobase: Regions with high levels of coverage have low levels of the modified base and vice versa. The mean coverage of post-digestion Excision-seq data for mapping uracil in dut1-1 ung1 $1 \Delta$ yeast was 8.2 reads per base, with a standard deviation of 6.8. With respect to replication timing, there was a 15fold difference in Excision-seq data coverage between early-firing origins and other regions $\sim 10 \mathrm{~kb}$ away (e.g., origin ARS428) (Fig. 3E). In contrast, in late-replicating regions, there was a sevenfold difference between the latest replicating DNA and nearby regions (e.g., peaks between ARS406 and 409) (Fig. 3A). Because post-digestion Excision-seq maintains strand information (Fig. 1B), we examined uracil content in leading and lagging strands. We selected 50 earlyfiring origins (Raghuraman et al. 2001) and examined the uracil content of leading and lagging strands within $2 \mathrm{~kb}$ of the ARS consensus sequence. We found that sequence coverage of the lagging strand in post-digestion Excision-seq data was $\sim 1$.3-fold higher than the leading strand, suggesting a bias toward higher uracil incorporation in the leading strand relative to the lagging strand during early replication (Fig. 3D) and possibly implicating a bias in uracil incorporation by DNA polymerases or pool availability.
We used Excision-seq to map uracil content in ung $1 \Delta$ yeast during pharmacological inhibition of thymidylate synthase with 5-fluorouracil (5-FU) (Seiple et al. 2006). Genomic DNA from ung1 $1 \Delta$ yeast treated with 5-FU had higher levels of overall uracil incorporation (Fig. 3A, green) but showed uracil depletion at early-firing origins of replication (Fig. 3A, ARS418 and ARS428), suggesting that early thymidylate synthesis is less sensitive to 5-FU treatment. The lack of uracil incorporation that we observed in latereplicating regions of the genome (Fig. $3 \mathrm{~A}$, red and blue) was not present in these data, suggesting that 5 -FU might activate a checkpoint, preventing late origins from firing (Santocanale and Diffley 1998; Feng et al. 2006; Seiple et al. 2006).

To discern global features of uracil Excision-seq data, we used a previously developed segmentation approach based on dynamic Bayesian networks (Hoffman et al. 2012) to identify correlations between uracil incorporation and other functional features of the genome. We segmented the yeast genome using uracil Excision-seq data and replication timing data (Raghuraman et al. 2001) to identify coherent patterns between these data sets (Fig. 3A). This analysis identified two major classes of genomic regions showing uracil depletion: those that replicate earliest and latest. At early-firing origins (e.g., Fig. 3A, ARS428 and label 7 in the segmentation), uracil content is low, centered on the site of Orc1 binding (Eaton et al. 2010), and uracil levels slowly increase at sites upstream of and downstream from the origin, similar to the phenomenon observed in E. coli (Fig. 1D). However, the latest replicating regions of the chromosome also exhibit uracil depletion (Fig. 3A, label 1 in the segmentation): The region between two early-firing origins (ARS418 and ARS428) exhibits variation in uracil incorporation that correlates with latereplicating regions. However, despite uracil depletion in late-replicating regions, the extent of depletion is reduced relative to early-firing origins (Fig. 3B, cf. peaks over ARS418 and ARS428 with intervening, late-replicating regions). To identify other genomic features that might correlate with genomic uracil content, we performed other segmentations, including DNase I hypersensitivity mapping data (Hesselberth et al. 2009) and mRNA expression levels measured by RNA-seq (Levin et al. 2010), but found that these signals did not qualitatively change the segmentations (data not shown), emphasizing that genomic uracil content correlates most strongly to replication timing.

\section{Application of Excision-seq to study pyrimidine dimers in DNA}

We extended Excision-seq to map two main classes of dipyrimidines caused by exposure to UV light: cyclobutane pyrimidine dimers (CPDs) and 6-4 photoproducts (6-4pp) (Pfeifer 1997). We 
A $1.5 \mathrm{Mb}$

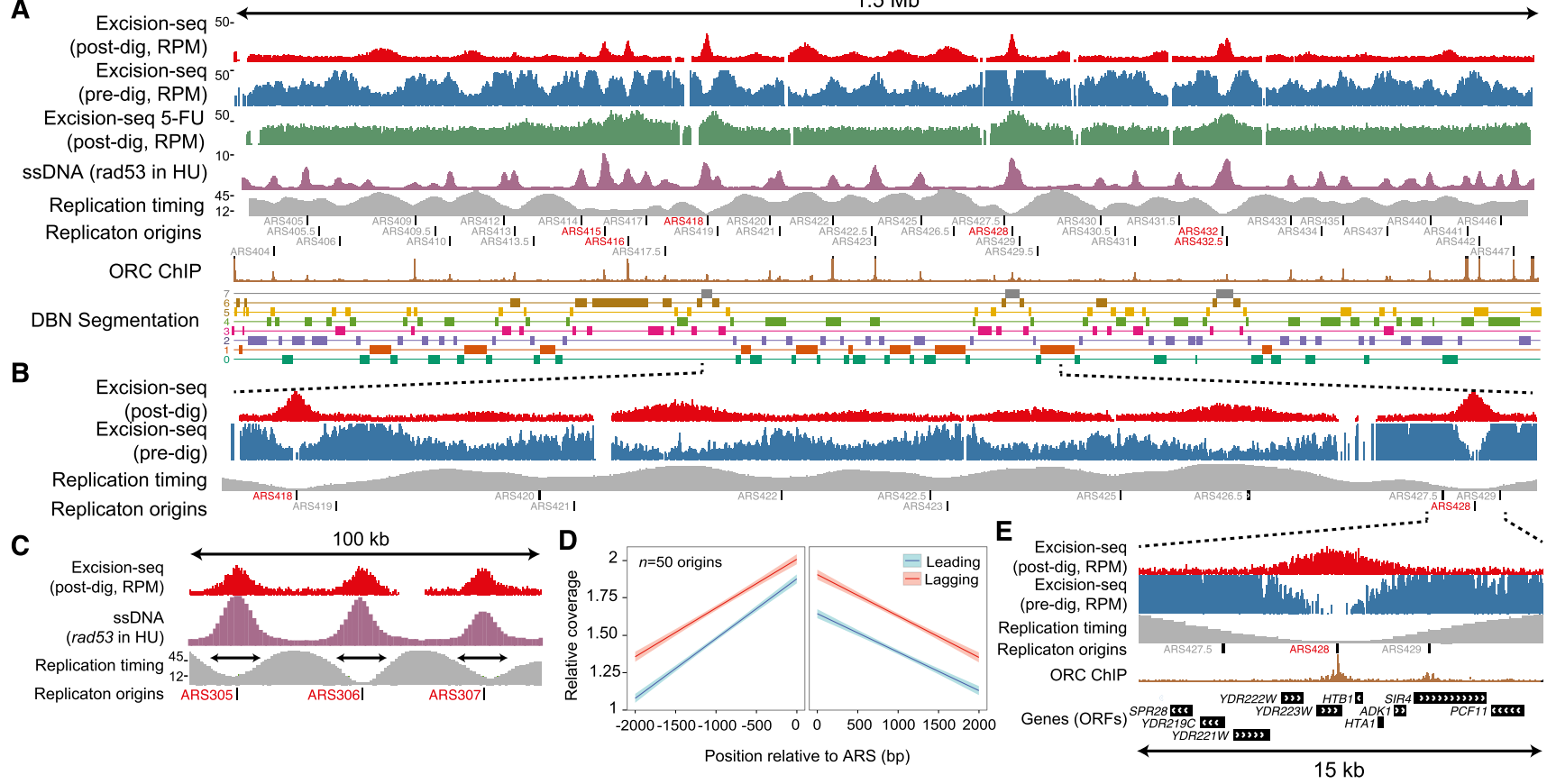

Figure 3. Excision-seq maps of uracil content in the budding yeast genome. (A) Data showing the entire yeast chromosome 4 for dut 1-1 ung $1 \Delta$ yeast using post-digestion Excision-seq (red, reads per million [RPM]), predigestion Excision-seq (blue, RPM), post-digestion Excision-seq data for ung1 $\Delta$ yeast treated with 5-fluorouracil (5-FU) (green, RPM), single-stranded DNA accumulation caused by hydroxyurea treatment of a rad53 yeast strain (purple, arbitrary units) (Feng et al. 2006), replication timing data ( $T_{\text {rep, }}$ minutes replicated after $\mathrm{G}_{1}$ release) (Raghuraman et al. 2001) (gray), annotated origins of replication (Nieduszynski et al. 2007), ORC chromatin immunoprecipitation signals (Eaton et al. 2010) (brown, coverage), and labeled segments from an eight-state DBN segmentation (Hoffman et al. 2012) incorporating replication timing (Yabuki et al. 2002) and post-digestion Excision-seq mapping of uracil. (B) A 450-kb region of chromosome 4 highlights patterns of uracil incorporation in early-replicating origins (ARS418 and ARS428), as well as uracil depletion in late-replicating regions. (C) Correspondence of peak widths between post-digestion Excision-seq (red) and ssDNA accumulation (Feng et al. 2006) (purple) at three early-replicating origins in a 100-kb region of chromosome 3. (D) Post-digestion Excision-seq measurement of uracil content for 50 earlyreplicating origins. Lagging strands have $\sim 1$.3-fold higher relative coverage than leading strands in post-digestion Excision-seq data, reflecting increased uracil content in leading strands. (E) A 15-kb region of chromosome 4 highlights patterns of uracil incorporation at the early-replicating origin ARS428.

collected high molecular weight genomic DNA from cells irradiated with UV light $\left(\sim 10,000 \mathrm{~J} / \mathrm{m}^{2} ;<10 \%\right.$ cell viability) and treated damaged DNA with $S$. pombe Uve1 (also known as UVDE), which recognizes and cleaves upstream of both CPDs and 6-4pp (Fig. 4A; Avery et al. 1999). Pyrimidine dimers created at the ends of DNA fragments upon UVDE digestion inhibited downstream polishing and adaptor ligation (data not shown), so we repaired 5' pyrimidine dimers in vitro to "mono" pyrimidines using photolyase enzymes that recognize either cyclobutane dimers or 6-4 photoproducts (Fig. 4A; Sancar and Sancar 2006). These repaired 5'-dipyrimidine ends are compatible with subsequent polishing, adaptor ligation, and PCR. DNA sequences from these libraries were collected and aligned to the budding yeast genome to determine the dipyrimidine at their $5^{\prime}$ ends. More than $85 \%$ of the aligned sequences acquired from libraries prepared by treatment with CPD and 6-4pp photolyases derived from genomic positions with pyrimidine dimers-validating the method. In total, we identified 1,249,684 sites of CPD formation (38\% of all genomic dipyrimidines; $72 \%$ of TT) and 107,490 sites of 6-4pp formation (5\% of all genomic dipyrimidines). Dipyrimidine content of sequences reflected known photolyase specificities, recapitulating the expected distribution of CPDs (i.e., cyclobutane dimer prevalence is greatest for TT dipyrimidines, followed by TC, CT, and CC) and 6-4 photoproducts (i.e., TC dinucleotides are most abundant, followed by TT, CC, and CT) (Fig. 4B; Douki and Cadet 2001).

We examined local base content near the sites of linker attachment in the CPD and 6-4pp Excision-seq libraries (Fig. 4C,D). In the CPD library, $60 \%$ of the reads began with TT dinucleotides, followed by CT dinucleotides (15\% of reads) (Fig. 4B). In the CPD library, normalized nucleotide frequencies upstream of and downstream from the first and second positions were not skewed relative to genomic frequencies of the budding yeast genome (Fig. 4C). For the 6-4pp libraries, the first and second positions of the reads exhibited a strong bias toward pyrimidines. Notably, the base downstream from the dipyrimidine in the 6-4pp libraries was most often an A residue (with TCA and TTA comprising $~ 20 \%$ of the total reads), suggesting that $6-4$ photoproducts are preferentially created at these trinucleotides, or that the $X$. laevis 6-4 photolyase enzyme preferentially repairs these sites. This bias is not likely due to the UVDE enzyme, as it was also used to prepare the CPD libraries, which did not have detectable bias toward any residue at the position downstream from the dipyrimidine (Fig. 4D).

\section{Discussion}

Application of Excision-seq to study uracil content in DNA revealed previously unknown variation in uracil content that is highly correlated with DNA replicating timing. We hypothesize that variation in uracil content is established by changes in dNTP pool composition such that the pool of nucleotides available for early and late replication contains higher levels of TTP than dUTP. Comparison of our data to previous studies that measured the levels of single-stranded DNA accumulation following depletion of dNTPs with hydroxyurea (HU) (Feng et al. 2006) revealed that the regions of depleted uracil content (e.g., post-digestion Excision-seq 
A

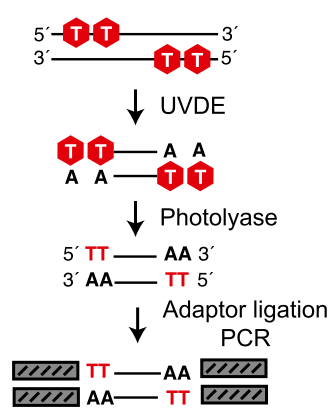

C

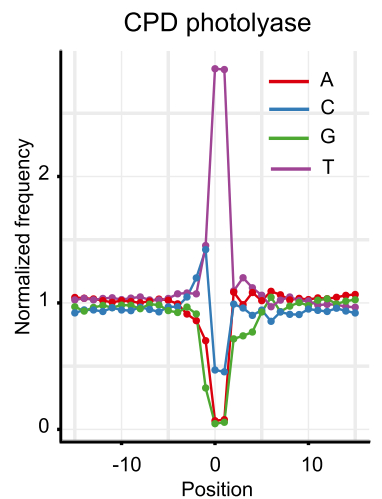

B

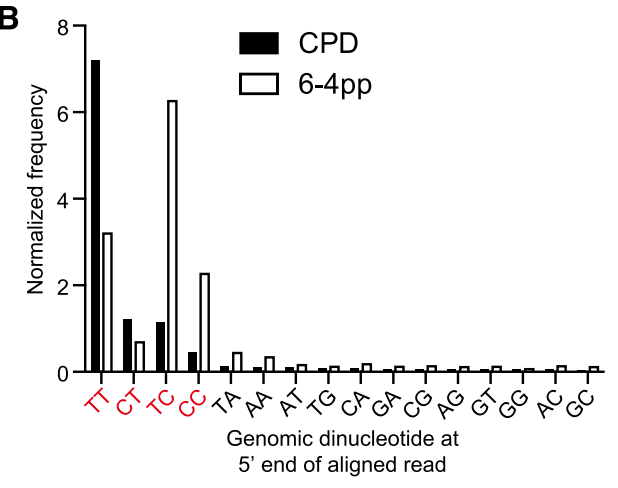

D

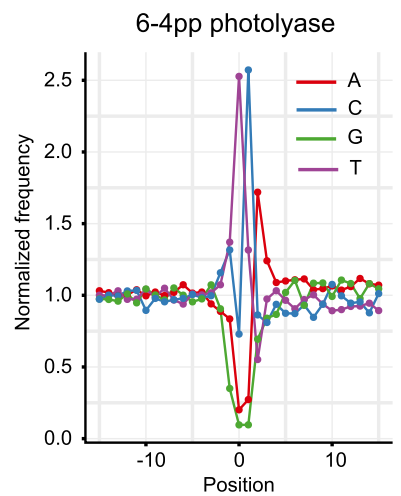

Figure 4. Excision-seq mapping of dipyrimidines in the budding yeast genome. $(A)$ In predigestion Excision-seq for pyrimidine dimers, UV-damaged DNA is cleaved with UVDE, releasing double-stranded fragments with five dipyrimidines (red). Fragments are treated with CPD or 6-4pp photolyase enzymes, repairing dipyrimidines to "mono" pyrimidines, and yielding ends compatible with polishing, ligation, and PCR. (B) Analysis of sequencing libraries treated with CPD or 6-4pp photolyase prepared from UVirradiated DNA showed an enrichment of sequence reads with dipyrimidine ends (red text) relative to genomic dinucleotide content and recapitulated the known specificity of the photolyase enzymes (Chowdhury and Guengerich 2008). (C) Frequency of nucleotides relative to mapped positions of sequences from predigestion Excision-seq libraries for mapping cyclobutane dimers in S. cerevisiae. Position 0 corresponds to the mapped position of the $5^{\prime}$ end of 5,063,196 sequencing reads. (D) Frequency of nucleotides relative to mapped positions of sequences from predigestion Excision-seq libraries for mapping 6-4 photoproducts in S. cerevisiae. Position 0 corresponds to the mapped position of the 5 ' end of $3,655,251$ sequencing reads.

peaks) are remarkably similar to the amount of DNA replicated from early-firing origins in the presence of HU (Fig. 3C). This correlation suggests that these two phenomena-dNTP depletion and TTP:dUTP equilibration-may happen at a similar time during $S$ phase. Recent studies also showed that dNTP pools synthesized during $\mathrm{G}_{1} / \mathrm{S}$ are limiting for DNA synthesis, allowing $\sim 5 \mathrm{~kb}$ of DNA to be replicated before a critical transition during replication (Poli et al. 2012). Together, these and our data suggest that dNTPs made during $\mathrm{G}_{1}$ (Koç et al. 2004) may be compositionally pure of dUTP, and the transition from low to high uracil content (e.g., summit to shoulder of Excision-seq peaks) (Fig. 3C) reflects equilibration of dUTP and TTP levels. Notably, the phenomenon of uracil content variation may enable simplified mapping of replication timing, as $u n g 1 \Delta$ yeast strains are readily created and human cell lines expressing a bacteriophage uracil DNA glycosylase inhibitor have undetectable levels of nuclear human UNG (also known as UNG1 and UNG2) activity (Weil et al. 2013).

The depletion of uracil in late-replicating regions found in uracil Excision-seq data is unexpected and suggests that dUTP availability is also reduced toward the end of replication. The dUMP substrate of thymidylate synthase is produced either by conversion of dUTP to
dUMP by dUTPase or by conversion of dCMP to dUMP by deoxycytidylate deaminase (Dcd1). We speculate that the TTP: dUTP ratio is high during early and late replication due to limited production of dUTP (e.g., less ribonucleotide reductasemediated reduction of UDP), while TTP levels are maintained by the activity of dCMP deaminase.

One prediction of uracil content variation is that the mutational signature of uracil incorporation might correlate with DNA replication timing, possibly over evolutionary time scales. In budding yeast, Ung1 action on A: $U$ base pairs produces abasic sites in the template strand that are copied by the error-prone Rev1/Rev3 translesion polymerase, which incorporates a $\mathrm{C}$ across from the abasic site, yielding an A-to-C transversion (Collura et al. 2012). However, these diagnostic A:C transversions were not correlated with replication timing in yeast (Agier and Fischer 2012) or humans (Stamatoyannopoulos et al. 2009), suggesting that uracil excision repair is highly efficient under physiological conditions, limiting uracilmediated mutational signatures.

Variation in uracil content protects large chromosomal regions containing replication origins from uracil incorporation. If a similar mechanism for uracil content variation operates in human cells, it might inherently counteract common chemotherapies such as 5-fluorouracil, which raise dUTP levels to promote cycles of incorporation and repair, causing DNA damage and apoptosis. Elucidation of the mechanism underlying uracil content variation may therefore have implications for the design and delivery of therapeutics that cause nucleotide pool imbalances to promote cell death.

Unlike uracil incorporation, CPD and 6-4pp dimers were uniformly distributed across the genome, consistent with previous studies showing uniform densities of CPD formation (Teng et al. 2011). Future application of Excision-seq to map UV photoproducts may uncover more subtle patterns associated with genomic features, including nucleosome positions or other structural features. These studies could provide insight into the nature of mutational hotspots found in DNA preferentially caused by 6-4 photoproducts, which are more mutagenic than CPD photoproducts (Mitchell 1988). Application of Excision-seq in mammalian cells would enable the study of photoproduct formation in the context of methylated CpG dinucleotides, which are prone to mutation (You et al. 1999).

Analysis of Excision-seq signals for the mapping of uracil and pyrimidine dimers enables an estimation of the specificity of the Excision-seq method. Nearly $98 \%$ of the reads from the uracil predigestion libraries map to positions downstream from a $T$ residue (i.e., uracil in the DNA) (Fig. 2A), demonstrating the high specificity of UDG and consistent with previous measurement of its specificity for uracil in vitro (Stivers et al. 1999). Similar measurements of the UVDE enzyme show that it efficiently recognizes and cleaves at 
pyrimidine dimers but also recognizes a variety of other nucleobase lesions in DNA (Avery et al. 1999). The specificity for dipyrimidines is recapitulated in the CPD and 6-4pp Excision-seq libraries, where many of the reads begin with dipyrimidines (Fig. 4B). These data suggest that the specificity of Excision-seq is largely dictated by the specificity of excision repair enzymes. Based on its recovery of expected sites of modification, Excision-seq has a low empirical false positive rate for individual sites of uracil modification ( $2 \%$ for uracil predigestion libraries). For the CPD and 6-4pp libraries, 93\% and $86 \%$ of the sequences began with dipyrimidines, initially suggesting a high false positive rate ( $7 \%$ and $14 \%$, respectively). However, signals from the CPD and 6-4pp libraries could also be attributed to the extended ability of UVDE to cleave at other types of bulky DNA lesions (Avery et al. 1999). Notably, AA and TA were the most abundant dinucleotides other than dipyrimidines in both CPD and 6-4pp libraries, with AA dinucleotides at the beginning of $4 \%$ of the reads for both libraries and TA dinucleotides beginning $3 \%$ of reads for CPD libraries. Little is known about photoproducts involving purines (Pfeifer 1997), but previous studies detected photoproduct formation in synthetic TA and AA dinucleotides (Bose et al. 1983; Gallagher and Duker 1986), consistent with signals we observe from photoproducts formed in genomic DNA. It is possible that the AA and TA dinucleotides present in the CPD and 6-4pp libraries more accurately reflect the UV-induced formation of photoproducts at genomic dinucleotides involving purines, or these could be false positive signals generated by, e.g., photolyase bias in the assay. Finally, other factors including the quality of DNA starting material for Excision-seq libraries could influence the false positive rate for individual samples. For example, predigestion Excision-seq libraries should be constructed with carefully prepared high molecular weight DNA to reduce the amount of nonspecific fragmentation, which would be captured in a sequencing library (Fig. 1A).

The sensitivity of the Excision-seq method can also be considered. In predigestion Excision-seq, the ability to capture low levels of modified nucleobases is influenced by the efficiency of the excision repair enzyme and sufficient levels of modified nucleobases to enable creation of double-stranded DNA fragments. We found that shearing of DNA from dut1-1 ung1 $1 \Delta$ yeast by UDG was not enhanced by extended incubation times (data not shown), indicating quantitative cleavage of uracil bases. The sensitivity of post-digestion Excision-seq is dictated by the overall incorporation levels of modified nucleobases at the same position in a population of molecules. In the future, the combination of post-digestion Excision-seq with single molecule tagging strategies should enable more precise quantitation of modified nucleobase incorporation in large genomes (Schmitt et al. 2012; Hiatt et al. 2013).

The availability of a variety of excision repair enzymes will facilitate the study of other modifications by Excision-seq. Many cell intrinsic (e.g., inosine) and extrinsic (e.g., 8-oxo-guanine) modified nucleobases have cognate repair enzymes, possibly enabling their study by Excision-seq (Tchou et al. 1994; Saparbaev et al. 2000). The removal of oxidized cytosine bases during DNA demethylation is catalyzed by the base excision repair enzyme TDG, which could be used in Excision-seq to generate maps of oxidized cytosine bases in mammalian DNA (Kohli and Zhang 2013). Finally, ribonucleotide incorporation into DNA could also be studied by Excision-seq using Ribonuclease HII enzymes to cleave at sites of ribonucleotide incorporation (Nick McElhinny et al. 2010).

Excision-seq enables the analysis of primary DNA modification events independently from fixed sequence polymorphisms, facilitating study of the mutational process. Large-scale surveys of cancer genomes have identified mutational signatures attributable to environmental exposure or dysregulated cellular physiology (Alexandrov et al. 2013). The Excision-seq method will be useful in generating high resolution global maps of DNA modification that can be integrated with surveys of DNA sequence polymorphisms (The 1000 Genomes Project Consortium 2012) and functional chromatin states (The ENCODE Project Consortium 2012) to provide insight into how DNA modifications are formed, how repair processes facilitate their conversion to mutation, and how chromatin context influences both DNA modification and repair.

\section{Methods}

\section{Strains and oligonucleotides}

Strains and oligonucleotides are available in Supplemental Methods.

\section{Excision-seq library methods}

Detailed methods for constructing Excision-seq libraries are available in Supplemental Methods. Briefly, in predigestion Excision-seq, DNA containing modified nucleobases was treated with excision repair enzymes, and adaptors were ligated to the sites of cleavage, facilitating PCR amplification of these fragments. In post-digestion Excision-seq, libraries of mechanically sheared genomic DNA were treated with excision repair enzymes to destroy strands containing the modified base, preventing their PCR amplification. Libraries were sequenced on Illumina MiSeq or HiSeq 2000 platforms using standard protocols.

\section{Analysis of Excision-seq data}

Sequences were analyzed by alignment to a reference genome (sacCer1) using Bowtie 2 (Langmead et al. 2009) and SAMtools (Li et al. 2009), processed to bedGraph format using BEDTools (Quinlan and Hall 2010), and visualized in the UCSC Genome Browser (Karolchik et al. 2011). Coverage at each position was normalized by the number of reads aligned in the library (i.e., reads per million $[\mathrm{RPM}])$. Using this method, the level of coverage at a specific site or region in the genome represents the relative quantity of modified base at that position. For Excision-seq libraries mapping dipyrimidines, dinucleotide counts for the $5^{\prime}$ ends of the reads were determined, and the frequencies of dinucleotide combinations were normalized to background frequencies found in S. cerevisiae genomic DNA to account for the A:T bias in the genome. Software and pipelines used to analyze data are available on GitHub (https:// github.com/hesselberthlab/modmap).

\section{DBN segmentation of genomic data sets}

We applied a segmentation approach using dynamic Bayesian networks (Hoffman et al. 2012) to find correlations between uracil content and replication timing (Raghuraman et al. 2001; Yabuki et al. 2002). An eight-state model was trained on $1 \%$ of the genome using a resolution of $500 \mathrm{bp}$, and the parameterized model was used to decode the rest of the genome.

\section{Data access}

Raw and processed sequencing data (FASTQ and bedGraph formats) from this study have been submitted to the NCBI Gene Expression Omnibus (GEO; http://www.ncbi.nlm.nih.gov/geo/) (Barrett et al. 2010) under accession number GSE51361.

\section{Acknowledgments}

We thank S. Fields, M. Huang, M. Johnston, and R. Sclafani for discussions and comments on the manuscript. We thank A. Sancar 
and D. Zhang for providing purified photolyase enzymes; P. Doetsch for the $S$. pombe UVDE expression plasmid; S. Boiteux for yeast strains; and M. Hoffman for advice on genome segmentations. This work was supported in part by a March of Dimes Basil O'Connor Starter Scholar award (J.R.H.), a Damon Runyon-Rachleff Innovation award (J.R.H.), a Research Scholar grant from the American Cancer Society (J.R.H., RSG-13-216-01-DMC), a Department of Defense Visionary Postdoc award (M.R., W81XWH-12-1-0333), and an NIH Molecular Biology Training grant (D.S.B., T32 GM008730).

\section{References}

The 1000 Genomes Project Consortium. 2012. An integrated map of genetic variation from 1,092 human genomes. Nature 491: 56-65.

Agier N, Fischer G. 2012. The mutational profile of the yeast genome is shaped by replication. Mol Biol Evol 29: 905-913.

Alexandrov LB, Nik-Zainal S, Wedge DC, Aparicio SAJR, Behjati S, Biankin AV, Bignell GR, Bolli N, Borg A, Børresen-Dale AL, et al. 2013. Signatures of mutational processes in human cancer. Nature 500: 415-421.

Avery AM, Kaur B, Taylor JS, Mello JA, Essigmann JM, Doetsch PW. 1999. Substrate specificity of ultraviolet DNA endonuclease (UVDE/Uve1p) from Schizosaccharomyces pombe. Nucleic Acids Res 27: 2256-2264.

Barrett T, Troup DB, Wilhite SE, Ledoux P, Evangelista C, Kim IF, Tomashevsky M, Marshall KA, Phillippy KH, Sherman PM, et al. 2010. NCBI GEO: archive for functional genomics data sets-10 years on. Nucleic Acids Res 39: D1005-D1010.

Besaratinia A, Pfeifer GP. 2012. Measuring the formation and repair of UV damage at the DNA sequence level by ligation-mediated PCR. Methods Mol Biol 920: 189-202.

Blount BC, Mack MM, Wehr CM, MacGregor JT, Hiatt RA, Wang G, Wickramasinghe SN, Everson RB, Ames BN. 1997. Folate deficiency causes uracil misincorporation into human DNA and chromosome breakage: implications for cancer and neuronal damage. Proc Natl Acad Sci 94: 3290-3295.

Bose SN, Davies RJ, Sethi SK, McCloskey JA. 1983. Formation of an adeninethymine photoadduct in the deoxydinucleoside monophosphate $\mathrm{d}(\mathrm{TpA})$ and in DNA. Science 220: 723-725.

Chowdhury G, Guengerich FP. 2008. Direct detection and mapping of sites of base modification in DNA fragments by tandem mass spectrometry. Agnew Chem Int Ed Engl 47: 381-384.

Clark TA, Spittle KE, Turner SW, Korlach J. 2011. Direct detection and sequencing of damaged DNA bases. Genome Integr 2: 10.

Clarke J, Wu HC, Jayasinghe L, Patel A, Reid S, Bayley H. 2009. Continuous base identification for single-molecule nanopore DNA sequencing. Nat Nanotechnol 4: 265-270.

Collura A, Kemp PAVD, Boiteux S. 2012. Abasic sites linked to dUTP incorporation in DNA are a major cause of spontaneous mutations in absence of base excision repair and Rad17-Mec3-Ddc1 (9-1-1) DNA damage checkpoint clamp in Saccharomyces cerevisiae. DNA Repair (Amst) 11: 294-303.

Dengg M, Garcia-Muse T, Gill SG, Ashcroft N, Boulton SJ, Nilsen H. 2006. Abrogation of the CLK-2 checkpoint leads to tolerance to base-excision repair intermediates. EMBO Rep 7: 1046-1051.

Douki T, Cadet J. 2001. Individual determination of the yield of the main UV-induced dimeric pyrimidine photoproducts in DNA suggests a high mutagenicity of CC photolesions. Biochemistry 40: 2495-2501.

Eaton ML, Galani K, Kang S, Bell SP, MacAlpine DM. 2010. Conserved nucleosome positioning defines replication origins. Genes Dev 24: 748753.

The ENCODE Project Consortium. 2012. An integrated encyclopedia of DNA elements in the human genome. Nature 489: 57-74.

Feng W, Collingwood D, Boeck ME, Fox LA, Alvino GM, Fangman WL, Raghuraman MK, Brewer BJ. 2006. Genomic mapping of single-stranded DNA in hydroxyurea-challenged yeasts identifies origins of replication. Nat Cell Biol 8: 148-155.

Friedberg EC. 2001. How nucleotide excision repair protects against cancer. Nat Rev Cancer 1: 22-33.

Gadsden MH, McIntosh EM, Game JC, Wilson PJ, Haynes RH. 1993. dUTP pyrophosphatase is an essential enzyme in Saccharomyces cerevisiae. EMBO J 12: 4425-4431.

Gallagher PE, Duker NJ. 1986. Detection of UV purine photoproducts in a defined sequence of human DNA. Mol Cell Biol 6: 707-709.

Guillet M, van der Kemp PA, Boiteux S. 2006. dUTPase activity is critical to maintain genetic stability in Saccharomyces cerevisiae. Nucleic Acids Res 34: 2056-2066.

Hanawalt PC, Spivak G. 2008. Transcription-coupled DNA repair: two decades of progress and surprises. Nat Rev Mol Cell Biol 9: 958-970.
Hesselberth JR, Chen X, Zhang Z, Sabo PJ, Sandstrom R, Reynolds AP, Thurman RE, Neph S, Kuehn MS, Noble WS, et al. 2009. Global mapping of protein-DNA interactions in vivo by digital genomic footprinting. Nat Methods 6: 283-289.

Hiatt JB, Pritchard CC, Salipante SJ, O'Roak BJ, Shendure J. 2013. Single molecule molecular inversion probes for targeted, high-accuracy detection of low-frequency variation. Genome Res 23: 843-854.

Hoffman MM, Buske OJ, Wang J, Weng Z, Bilmes JA, Noble WS. 2012. Unsupervised pattern discovery in human chromatin structure through genomic segmentation. Nat Methods 9: 473-476.

Karolchik D, Hinrichs AS, Kent WJ. 2011. The UCSC Genome Browser. Curr Protoc Hum Genet 71: 18.6.1-18.6.33.

Kavli B, Otterlei M, Slupphaug G, Krokan HE. 2007. Uracil in DNA-general mutagen, but normal intermediate in acquired immunity. DNA Repair (Amst) 6: 505-516.

Kim N, Jinks-Robertson S. 2009. dUTP incorporation into genomic DNA is linked to transcription in yeast. Nature 459: 1150-1153.

Klemm L, Duy C, Iacobucci I, Kuchen S, von Levetzow G, Feldhahn N, Henke N, Li Z, Hoffmann TK, Kim YM, et al. 2009. The B cell mutator AID promotes B lymphoid blast crisis and drug resistance in chronic myeloid leukemia. Cancer Cell 16: 232-245.

Koç A, Wheeler LJ, Mathews CK, Merrill GF. 2004. Hydroxyurea arrests DNA replication by a mechanism that preserves basal dNTP pools. J Biol Chem 279: 223-230.

Kohli RM, Zhang Y. 2013. TET enzymes, TDG and the dynamics of DNA demethylation. Nature 502: 472-479.

Kozdon JB, Melfi MD, Luong K, Boitano M, Wang S, Zhou B, Gonzalez D, Collier J, Shapiro L, McAdams HH. 2013. Global methylation state at base-pair resolution of the Caulobacter genome throughout the cell cycle. Proc Natl Acad Sci 110: E4658-E4667.

Krokan HE, Drabløs F, Slupphaug G. 2002. Uracil in DNA-occurrence, consequences and repair. Oncogene 21: 8935-8948.

Langmead B, Trapnell C, Pop M, Salzberg SL. 2009. Ultrafast and memoryefficient alignment of short DNA sequences to the human genome. Genome Biol 10: R25.

Law YK, Forties RA, Liu X, Poirier MG, Kohler B. 2013. Sequence-dependent thymine dimer formation and photoreversal rates in double-stranded DNA. Photochem Photobiol Sci 12: 1431-1439.

Levin JZ, Yassour M, Adiconis X, Nusbaum C, Thompson DA, Friedman N, Gnirke A, Regev A. 2010. Comprehensive comparative analysis of strand-specific RNA sequencing methods. Nat Methods 7: 709-715.

Li H, Handsaker B, Wysoker A, Fennell T, Ruan J, Homer N, Marth G, Abecasis G, Durbin R, 1000 Genome Project Data Processing Subgroup. 2009. The Sequence Alignment/Map format and SAMtools. Bioinformatics 25: 2078-2079.

Longley DB, Harkin DP, Johnston PG. 2003. 5-fluorouracil: mechanisms of action and clinical strategies. Nat Rev Cancer 3: 330-338.

Maul RW, Gearhart PJ. 2010. AID and somatic hypermutation. Adv Immunol 105: 159-191.

Maul RW, Saribasak H, Martomo SA, McClure RL, Yang W, Vaisman A, Gramlich HS, Schatz DG, Woodgate R, Wilson DM, et al. 2011. Uracil residues dependent on the deaminase AID in immunoglobulin gene variable and switch regions. Nat Immunol 12: 70-76.

Mitchell DL. 1988. The relative cytotoxicity of (6-4) photoproducts and cyclobutane dimers in mammalian cells. Photochem Photobiol 48: 51-57.

Nick McElhinny SA, Watts BE, Kumar D, Watt DL, Lundström EB, Burgers PMJ, Johansson E, Chabes A, Kunkel TA. 2010. Abundant ribonucleotide incorporation into DNA by yeast replicative polymerases. Proc Natl Acad Sci 107: 4949-4954.

Nieduszynski CA, Hiraga SI, Ak P, Benham CJ, Donaldson AD. 2007. OriDB: a DNA replication origin database. Nucleic Acids Res 35: D40-D46.

Pfeifer GP. 1997. Formation and processing of UV photoproducts: effects of DNA sequence and chromatin environment. Photochem Photobiol 65: 270-283.

Pfeifer GP, Drouin R, Riggs AD, Holmquist GP. 1991. In vivo mapping of a DNA adduct at nucleotide resolution: detection of pyrimidine (6-4) pyrimidone photoproducts by ligation-mediated polymerase chain reaction. Proc Natl Acad Sci 88: 1374-1378.

Poli J, Tsaponina O, Crabbé L, Keszthelyi A, Pantesco V, Chabes A, Lengronne A, Pasero P. 2012. dNTP pools determine fork progression and origin usage under replication stress. EMBO J 31: 883-894.

Protić-Sabljić M, Tuteja N, Munson PJ, Hauser J, Kraemer KH, Dixon K. 1986. UV light-induced cyclobutane pyrimidine dimers are mutagenic in mammalian cells. Mol Cell Biol 6: 3349-3356.

Quinlan AR, Hall IM. 2010. BEDTools: a flexible suite of utilities for comparing genomic features. Bioinformatics 26: 841-842.

Raghuraman MK, Winzeler EA, Collingwood D, Hunt S, Wodicka L, Conway A, Lockhart DJ, Davis RW, Brewer BJ, Fangman WL. 2001. Replication dynamics of the yeast genome. Science 294: 115-121.

Sale JE. 2013. Translesion DNA synthesis and mutagenesis in eukaryotes. Cold Spring Harb Perspect Biol 5: a012708. 
Sancar GB, Sancar A. 2006. Purification and characterization of DNA photolyases. Methods Enzymol 408: 121-156.

Santocanale C, Diffley JF. 1998. A Mec1- and Rad53-dependent checkpoint controls late-firing origins of DNA replication. Nature 395: 615-618.

Saparbaev M, Mani JC, Laval J. 2000. Interactions of the human, rat, Saccharomyces cerevisiae and Escherichia coli 3-methyladenine-DNA glycosylases with DNA containing dIMP residues. Nucleic Acids Res 28: 1332-1339.

Schmitt MW, Kennedy SR, Salk JJ, Fox EJ, Hiatt JB, Loeb LA. 2012. Detection of ultra-rare mutations by next-generation sequencing. Proc Natl Acad Sci 109: $14508-14513$.

Schumacher A, Kapranov P, Kaminsky Z, Flanagan J, Assadzadeh A, Yau P, Virtanen C, Winegarden N, Cheng J, Gingeras T, et al. 2006. Microarraybased DNA methylation profiling: technology and applications. Nucleic Acids Res 34: 528-542.

Seiple L, Jaruga P, Dizdaroglu M, Stivers JT. 2006. Linking uracil base excision repair and 5-fluorouracil toxicity in yeast. Nucleic Acids Res 34: $140-151$.

Smerdon MJ, Conconi A. 1999. Modulation of DNA damage and DNA repair in chromatin. Prog Nucleic Acid Res Mol Biol 62: 227-255.

Stamatoyannopoulos JA, Adzhubei I, Thurman RE, Kryukov GV, Mirkin SM, Sunyaev SR. 2009. Human mutation rate associated with DNA replication timing. Nat Genet 41: 393-395.

Stivers JT, Pankiewicz KW, Watanabe KA. 1999. Kinetic mechanism of damage site recognition and uracil flipping by Escherichia coli uracil DNA glycosylase. Biochemistry 38: 952-963.

Sugasawa K. 2010. Regulation of damage recognition in mammalian global genomic nucleotide excision repair. Mutat Res 685: 29-37.

Tchou J, Bodepudi V, Shibutani S, Antoshechkin I, Miller J, Grollman AP, Johnson F. 1994. Substrate specificity of Fpg protein. Recognition and cleavage of oxidatively damaged DNA. J Biol Chem 269: 15318-15324.

Teng Y, Bennett M, Evans KE, Zhuang-Jackson H, Higgs A, Reed SH, Waters R. 2011. A novel method for the genome-wide high resolution analysis of DNA damage. Nucleic Acids Res 39: e10.
Törmänen VT, Pfeifer GP. 1992. Mapping of UV photoproducts within ras proto-oncogenes in UV-irradiated cells: correlation with mutations in human skin cancer. Oncogene 7: 1729-1736.

Tornaletti S, Rozek D, Pfeifer GP. 1994. Mapping of UV photoproducts along the human P53 gene. Ann NY Acad Sci 726: 324-326.

Warner HR, Duncan BK, Garrett C, Neuhard J. 1981. Synthesis and metabolism of uracil-containing deoxyribonucleic acid in Escherichia coli. J Bacteriol 145: 687-695.

Weil AF, Ghosh D, Zhou Y, Seiple L, McMahon MA, Spivak AM, Siliciano RF Stivers JT. 2013. Uracil DNA glycosylase initiates degradation of HIV-1 cDNA containing misincorporated dUTP and prevents viral integration. Proc Natl Acad Sci 110: E448-E457.

Wu H, Zhang Y. 2014. Reversing DNA methylation: mechanisms, genomics, and biological functions. Cell 156: 45-68.

Yabuki N, Terashima H, Kitada K. 2002. Mapping of early firing origins on a replication profile of budding yeast. Genes Cells 7: 781-789.

Yan XJ, Xu J, Gu ZH, Pan CM, Lu G, Shen Y, Shi JY, Zhu YM, Tang L, Zhang $\mathrm{XW}$, et al. 2011. Exome sequencing identifies somatic mutations of DNA methyltransferase gene DNMT3A in acute monocytic leukemia. Nat Genet 43: 309-315.

You YH, Li C, Pfeifer GP. 1999. Involvement of 5-methylcytosine in sunlight-induced mutagenesis. J Mol Biol 293: 493-503.

You YH, Szabó PE, Pfeifer GP. 2000. Cyclobutane pyrimidine dimers form preferentially at the major $p 53$ mutational hotspot in UVB-induced mouse skin tumors. Carcinogenesis 21: 2113-2117.

Zavala AG, Morris RT, Wyrick JJ, Smerdon MJ. 2014. High-resolution characterization of CPD hotspot formation in human fibroblasts. Nucleic Acids Res 42: 893-905.

Zhou ZX, Zhang MJ, Peng X, Takayama Y, Xu XY, Huang LZ, Du LL. 2013. Mapping genomic hotspots of DNA damage by a single-strand-DNAcompatible and strand-specific ChIP-seq method. Genome Res 23: 705-715.

Received February 14, 2014; accepted in revised form July 8, 2014.

1542 Genome Research www.genome.org 


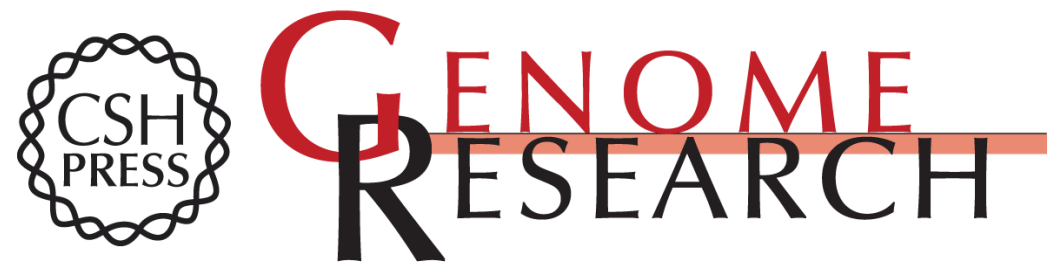

\section{High resolution mapping of modified DNA nucleobases using excision repair enzymes}

D. Suzi Bryan, Monica Ransom, Biniam Adane, et al.

Genome Res. 2014 24: 1534-1542 originally published online July 11, 2014 Access the most recent version at doi:10.1101/gr.174052.114

Supplemental Material

References

Open Access

Creative Commons License

Email Alerting Service
http://genome.cshlp.org/content/suppl/2014/07/14/gr.174052.114.DC1

This article cites 73 articles, 19 of which can be accessed free at: http://genome.cshlp.org/content/24/9/1534.full.html\#ref-list-1

Freely available online through the Genome Research Open Access option.

This article, published in Genome Research, is available under a Creative Commons License (Attribution 4.0 International), as described at http://creativecommons.org/licenses/by/4.0.

Receive free email alerts when new articles cite this article - sign up in the box at the top right corner of the article or click here.

\section{Affordable, Accurate Sequencing.}

